\title{
Seasonal Variations of the Near-Surface Atmospheric Boundary Layer Structure in China's Gurbantünggüt Desert
}

\author{
Ali Mamtimin $\mathbb{D}^{1,2}$ Yu Wang, ${ }^{1,2}$ Hajigul Sayit, ${ }^{3}$ XingHua Yang $\mathbb{D}^{1,2}$ Fan Yang, ${ }^{1,2}$ \\ Wen Huo $\mathbb{1}^{1},{ }^{1,2}$ and Chenglong Zhou ${ }^{1,2}$ \\ ${ }^{1}$ Taklimakan Desert Meteorology Field Experiment Station of CMA, Institute of Desert Meteorology, \\ China Meteorological Administration, Urumqi 830002, China \\ ${ }^{2}$ Center of Central Asia Atmospheric Science Research, Urumqi 830002, China \\ ${ }^{3}$ Newsroom of Xinjiang Meteorological Bureau, Urumqi 830002, China \\ Correspondence should be addressed to Wen Huo; huowenpet@idm.cn
}

Received 13 August 2019; Revised 7 January 2020; Accepted 31 January 2020; Published 28 February 2020

Academic Editor: Jorge E. Gonzalez

Copyright ( 2020 Ali Mamtimin et al. This is an open access article distributed under the Creative Commons Attribution License, which permits unrestricted use, distribution, and reproduction in any medium, provided the original work is properly cited.

\begin{abstract}
As the largest fixed and semifixed desert in China, the Gurbantünggüt Desert has a longperiod of snow in winter and the rapid growth of ephemeral plants in spring, presentingthe obvious seasonal changes in the underlying desert surface type, which could lead to the significantvariety in the near-surface boundary layer over this desert. To clarify the influence of the underlying surface change on the near-surface atmospheric boundary layer, gradient tower data and Eddy covariance data in 2017 were analyzed. The results were as follows: the wind profile can be divided into the nocturnal stable boundary layer and the daytime unstable boundary in spring, summer, and autumn, while the wind profile dominating nighttime stability in winter. During the study period, the four-season temperature profiles can be divided into four types: night radiation type, morning transition type, daylight solar radiation type, and evening transition type, and the temperature difference between spring and summer is more than that of autumn and winter. The vertical temperature lapse rate can reach $4.5^{\circ} \mathrm{C} / 100 \mathrm{~m}$ in spring and summer, while the vertical temperature lapse rate is $0.5^{\circ} \mathrm{C} / 100 \mathrm{~m}$ in winter. The special humidity value in summer and spring is greater than autumn and winter. The profile is almost in the inverse humidity state at almost all periods in winter. The inverse humidity phenomenon occurred on the autumn night. Besides, the specific humidity is closely related to the temperature and the near-surface wind speed. The "rapid change" of the underlying surface of the spring desert region affects the surface energy budget, which affects the turbulent energy and the stability of the near-surface layer, thus affecting the changes in temperature, humidity, and wind profile.
\end{abstract}

\section{Introduction}

The near-surface atmospheric boundary layer refers to a boundary layer that extends several meters above the ground (thicknesses can reach $50-100 \mathrm{~m}$ ) that is found in the atmospheric boundary layer [1]. This boundary layer can be directly affected by the underlying surface, and the changes in meteorological conditions can be viewed as direct manifestations of land-atmosphere interactions. Compared to the majority of the world's surface types, the desert covers the extensive spatial areas and has distinctly different surface properties. Therefore, deserts have a large influence on the climate and atmospheric circulation [2]. The desert areas have high surface temperatures, few clouds, dry surfaces, and high sensible heat fluxes, so the desert boundary layer is usually thicker [3]. The Gurbantünggüt Desert is the only desert in China where snow exists for a long time during the winter, and there is short-lived vegetation during the spring $[4,5]$. This unique desert environment is especially sensitive to climate changes, it follows a specific pattern in material, energy, and water circulation, and it plays an important part in influencing the region's climate.

Many researchers have studied the characteristics of the desert's boundary layer structure. Chen et al. [6] used a $20 \mathrm{~m}$ meteorological tower in the Zhurihe desert area to gather data for one year on the wind direction, wind speed, air 
temperature, and relative humidity and found the temperature rises rapidly, the wind speed is maximal, and the relative humidity is minimal in spring. Cuesta et al. [7] documented the seasonal evolution of the Saharan atmospheric boundary layer (SABL) by using a mobile multiplatform atmospheric observatory implemented in Tamanrasset (Algeria) in 2006. Observations showed a marked seasonal evolution of the SABL characteristics and a large variability during the West African monsoon onset phase. Cuesta et al. [8] revealed a variety of new dynamical mechanisms that control the structure of the SABL, which are responsible for exchange between the Saharan convective and residual boundary layers. Kalapureddy et al. [9] found a pronounced diurnal cycle of the African Easterly Jet, characterized by a decrease of wind speed during the afternoon. The interaction between the planetary boundary layer and mid-troposphere is likely responsible for this daily decrease of the African Easterly Jet. Garcia-Carreras et al. [10] described the turbulent structure and growth of the remote Saharan atmospheric boundary layer (ABL) by in situ radiosonde and aircraft measurements and a large Eddy simulation model. Messager et al. [11] utilized the radar sounding data of Algerian station: in Salah. During the African Monsoon Multidisciplinary Analysis Special Observing Periods, we discussed the atmospheric boundary layer structure of Salah. Gu et al. [12] analyzed the meteorological element profile of the 2 types of underlying surfaces in the Taklimakan Desert's northern border. The results show that the diurnal variation of wind speed changed more obviously in Xiaotang than Hade, and humidity and temperature change were closely related, and they were also related to the speed of the wind near the ground. Wang et al. [13] utilized the sounding data of the Taklimakan Desert hinterland and discussed the impacts of sand-dust and precipitation events on the near-surface atmospheric boundary layer structure. There have been few systematic observations and experimental studies performed on the near-surface layer meteorological elements of the fixed and semifixed desert hinterlands.

The objective of this study was to characterize the nearsurface boundary layer structure with a focus on how it evolves under changing surface conditions. The approach taken is to analyze 2017 in situ observations taken at China's largest fixed/semifixed desert hinterland. This study revealed the effects of the underlying surface condition changes on the near-surface boundary layer structure, most notably, the effects on the near-surface layer's temperature and humidity, and changes in the wind profile.

\section{Material and Methods}

2.1. Site. The Gurbantünggüt Desert is China's secondlargest desert and the largest fixed/semifixed desert. There is long-term snow cover during the winter, the vegetation is diverse, and the vegetation coverage is higher [5]. The desert landscape is varied, consisting mainly of various forms of longitudinal dunes, crescent moon-shaped dune ranges, and honeycomb dunes. Due to the influence of the northwest wind, the longitudinal dunes move in the northwest- southeast direction. From north to southeast, an arc transitioning phenomenon is visible, whose length goes from hundreds of meters to tens of kilometers, with a height of $10-25 \mathrm{~m}$, which reach up to $50-100 \mathrm{~m}$ [14]. Because the Gurbantünggüt Desert's long-term snow cover in the winter and precipitation conditions is higher than the Taklimakan Desert, its vegetation variety is more diverse. The plants with the greatest area distribution are Haloxylon ammodendron and Haloxylon persicum, whose area distribution is up to 1 million hectares. They are mainly distributed in the fixed dunes on the desert edges and the interhill sand.

2.2. Micrometeorological Measurements. In order to study the land-atmosphere interaction in the fixed/semifixed desert, the Institute of Desert Meteorology, China Meteorological Administration, established a land-atmosphericinteraction observation station in KE LA MEI LI area (hereafter KLML station) in the hinterland of Gurbantünggüt Desert in 2012 . The observational data forthis study were obtained from the KLML station. The KLML station $\left(45^{\circ} 14^{\prime} 08.19^{\prime \prime} \mathrm{N}, 87^{\circ} 35^{\prime} 23.79^{\prime \prime} \mathrm{E}, 531 \mathrm{~m}\right.$, Figure 1) includes the gradient tower observation system, Eddy covariance system, and solar radiation observation system.

The gradient tower observation system collected continuous data from a $35 \mathrm{~m}$ micrometeorological tower. The meteorological tower is equipped with slow response sensors as wind cup anemometers (Model 010C, Met One Instruments, Inc., USA), wind wanes (Model 020C, Met One Instruments, Inc., USA), and temperature and humidity sensor (Model HMP45C, VAISALA, Helsinki, Finland) to measure the wind speed, wind direction, temperature, and humidity at different arbitrary levels (0.5, $1,2,4,10,20$, and $32 \mathrm{~m})$. The barometric pressure sensor (Model PTB220, VAISALA, Helsinki, Finland) was mounted at $1 \mathrm{~m}$. The snow depth sensor (Model SR50A-L, Campbell Scientific, Logan, UT, USA) was mounted at $2 \mathrm{~m}$. The data from these sensors are recorded continuously by a data logger (Model CR1000, Campbell Scientific, Logan, UT, USA) at $1 \mathrm{~Hz}$ intervals and averaged at $10 \mathrm{~s}, 1 \mathrm{~min}$, $30 \mathrm{~min}$, and 1 hour intervals.

The solar radiation observation system was installed on the south side of the observation field. The solar radiation observation system includes a net radiometer (Model NR01, Hukseflux, Netherlands) mounted at $1.5 \mathrm{~m}$ above the ground, quantum sensor (Model LI190SB, Campbell Scientific, Logan, UT, USA) mounted at $1.5 \mathrm{~m}$ above the ground, and infrared temperature sensor (Model IRR-P, Campbell Scientific, Logan, UT, USA) mounted at $1.5 \mathrm{~m}$ above the ground. Soil temperature probes (Model 109L, Campbell Scientific, Logan, UT, USA) were set at depth of $0 \mathrm{~cm},-5 \mathrm{~cm},-10 \mathrm{~cm},-20 \mathrm{~cm}$, and $-40 \mathrm{~cm}$, soil moisture probes (Model CS616, Campbell Scientific, Logan, UT, USA) were set at depth of $-5 \mathrm{~cm},-10 \mathrm{~cm}$, $-20 \mathrm{~cm}$, - and $40 \mathrm{~cm}$, heat flux plates (Model HFP01, Hukseflux, Netherlands) were set at depth of $-5 \mathrm{~cm},-10 \mathrm{~cm}$, $-20 \mathrm{~cm}$, and $-40 \mathrm{~cm}$, and soil thermal conductivity sensor (Model TP01, Hukseflux, Netherlands) was set at depth $-10 \mathrm{~cm}$. The data from these sensors are recorded continuously by a data logger (Model CR3000, Campbell Scientific, 


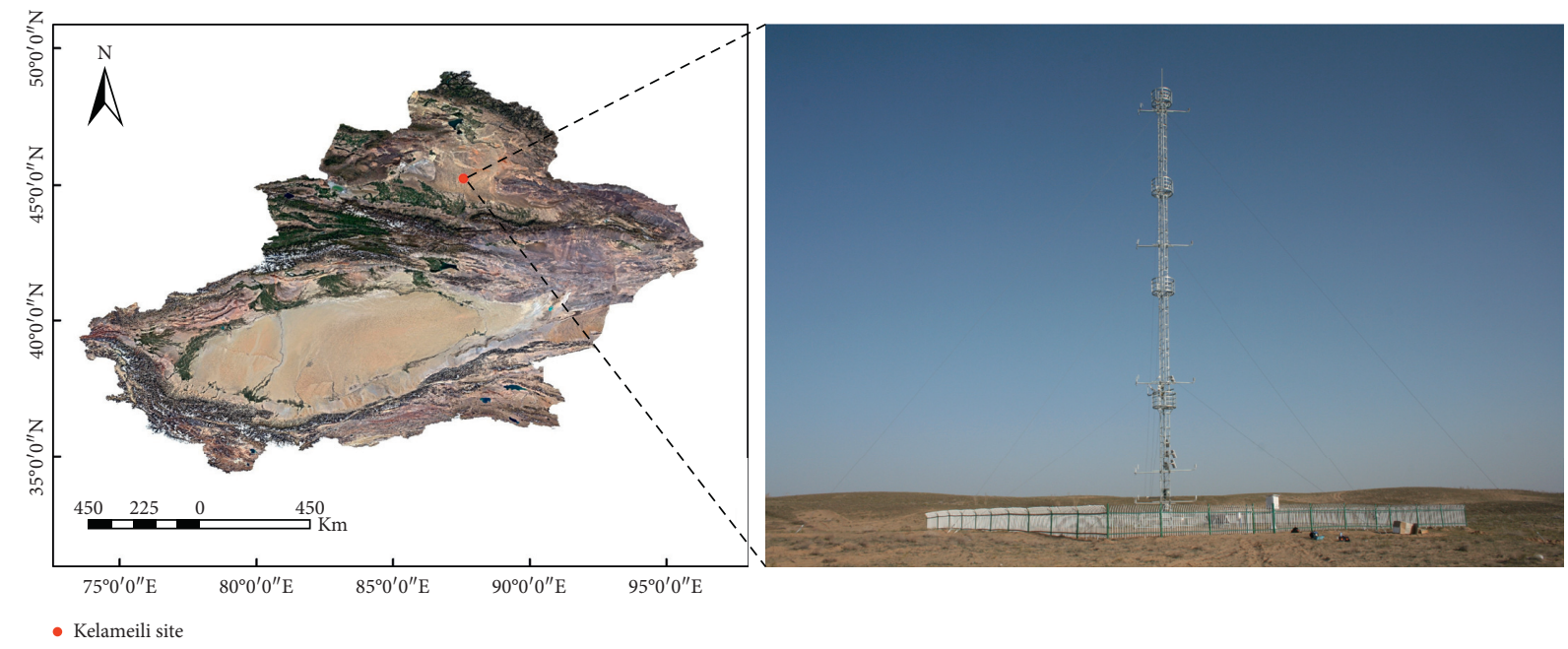

FIGURE 1: The study area and KLML land-atmospheric interaction observation station of the Gurbantünggüt Desert.

Logan, UT, USA) at $1 \mathrm{~Hz}$ intervals and averaged at $10 \mathrm{~s}, 1 \mathrm{~min}$, $30 \mathrm{~min}$, and 1 hour intervals.

The Eddy covariance system was mounted at $10 \mathrm{~m}$ of gradient tower. The Eddy covariance system (hereafter EC system) consists of an open-path infrared gas analyzer(Model Li7500A, Licor, Lincoln, NE, USA) and a 3D sonic anemometer(Model Gill Wind Master Pro, GILL Instruments Ltd., UK). The EC system sampled at $10 \mathrm{~Hz}$ by using theCR3000 datalogger (Model CR3000, Campbell Scientific,3 Logan, UT, USA), and raw data were stored for postprocessing. Postprocessing calculations were performed using EddyPro 6.0 software [14]. And the EddyPro 6.0 was used to process the raw data and generate $30 \mathrm{~min}$ flux data. We have applied a series of quality control for the use of EddyPro for calculating 30 min flux data such as spike count/ removal, amplitude resolution, dropouts, absolute limits, skewness and kurtosis, discontinuities, time lags, angle of attack, and steadiness of the horizontal wind. Tilt correction (DR method for coordinate rotation), time correction (maximum covariance with circular correlation), trend correction (block averaging), ultrasound virtual temperature correction, density correction (WPL revision), frequency response correction (analytic high-pass filtering, low-pass filtering), and assessment of data quality [23] were also carried out for processing the raw data.

The observation data used in this study include the 7 layer wind speed, temperature, and humidity data in the gradient observation system, which were taken from the observatory located in the hinterland of the Gurbantünggüt Desert. These data were observed in January, April, July, and October 2017. The data logger run in the local time ( UTC +5 hour 51 minutes) of the KLML station. Based on the results, between the $97^{\text {th }}$ and the $145^{\text {th }}$ day, the NDVI value of the Gurbantünggüt Desert increased rapidly, and it is part of the high-value phase of the year [5]. Based on climate data, January 2017 was a month with long-term snow cover. The annual herb plants bloomed much later, but they had a longer growth period. Thus, in the blooming summer months, there was maximum vegetation density [16]. In the present paper, we selected one-year meteorological observation data from gradient tower system to the analyzed the meteorological condition of the Gurbantünggüt Desert. About four seasons in the Gurbantünggüt Desert, we chose to study the typical underlying surfaces when there is desert snow, short-lived vegetation, and annual vegetation coverage to analyze the structural evolution characteristics of the near-surface boundary layer (Table 1). We chose March to October 2017 MODIS (Moderate Resolution Imaging Spectro-radiometer) level 3 standard data product, i.e., MOD13A3 (1 km horizontal resolution, monthly average, https://wist.echo.nasa.gov) to analyze NDVI variation which can indicate surface underlying change. The MOD13A3 data products are stored in ISIN (Integerized Sinusoidal), a global projection format. It divides the global image data into a 36column and 18-row grid. Each grid represents a storage area for file products. The position row and column number, such as h23v04 in the file name indicates the position of the 24th row and the 5th column. Xinjiang covers h23v04, h23v05, h24v04, h24v05, h25v04, and h25v056 regions, and KLML station is located in the h24v04 grid.

\section{Results and Discussion}

3.1. Meteorological Condition over the Gurbantünggüt Desert in 2017. The Gurbantünggüt Desert is a remote, arid, and rugged area, and it is separated by the Tian Shan mountains from the Ili River Basin, Turfan Depression, and the Tarim Basin of the southern Xinjiang. It is in this desert that the remotest point of land from any sea is located. The climate of the area is temperate, but very continental [16]. From the measured data of the KLML station in 2017, the annual variation curve of each meteorological element is shown in Figure 2. Figure 2(a) illustrates that the air temperature at the observation site in the hinterland of the Gurbantünggüt Desert is the lowest in January and the highest in July. The maximum temperature in the year is between -7.4 and $41.7^{\circ} \mathrm{C}$. The daily minimum temperature varies between -31 and $15.8^{\circ} \mathrm{C}$. In terms of a single day, the average daily temperature difference in winter, spring, summer, and autumn is about $10.3,11.3,12.4$, and $11.9^{\circ} \mathrm{C}$, respectively. Due 
TABLE 1: The sunny days of the four seasons in 2017.

\begin{tabular}{lc}
\hline Season & Sunny days \\
\hline Winter & January 1 to 5 , January 11 to 16 , January 18, January 22 to 27, and January 29 to 31 \\
Spring & April 1 to 3 , April 6 to 13 , April 15 to 16 , April 18 to 25 , and April 27 to 29 \\
Summer & July 1 to 14 , July 17 , July 19, and July 21 to 31 \\
Autumn & October 1 to 4 , October 6 to 18 , October 21 to 25 , and October 27 to 31
\end{tabular}

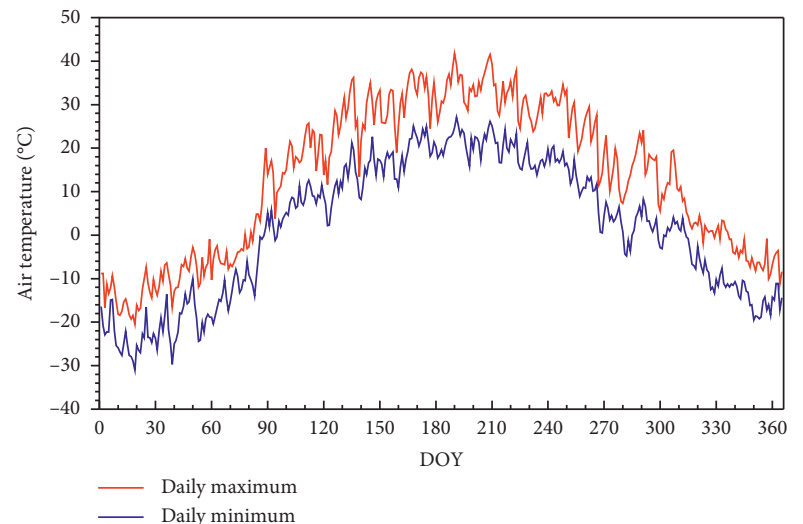

(a)

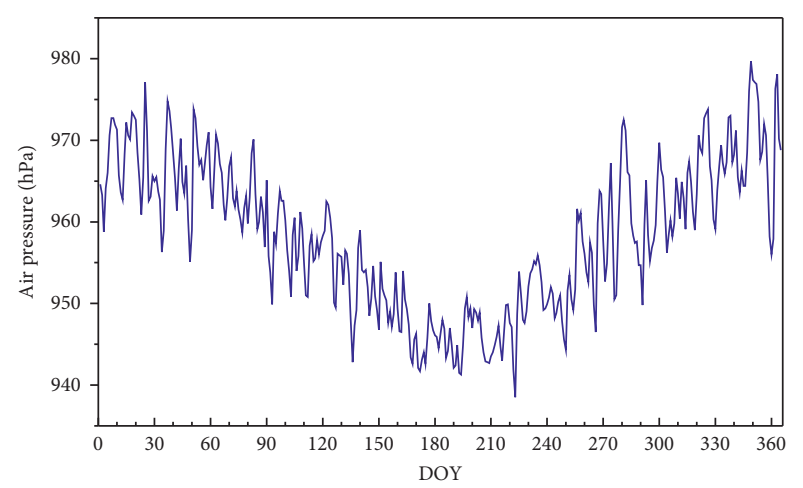

(c)

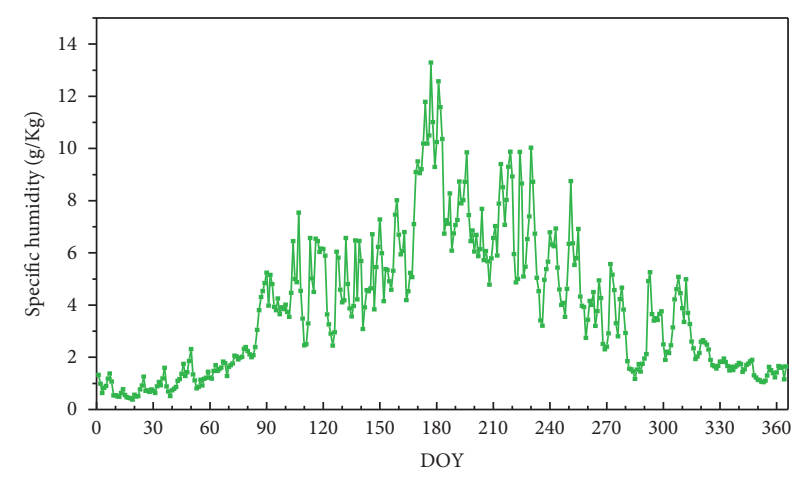

(b)

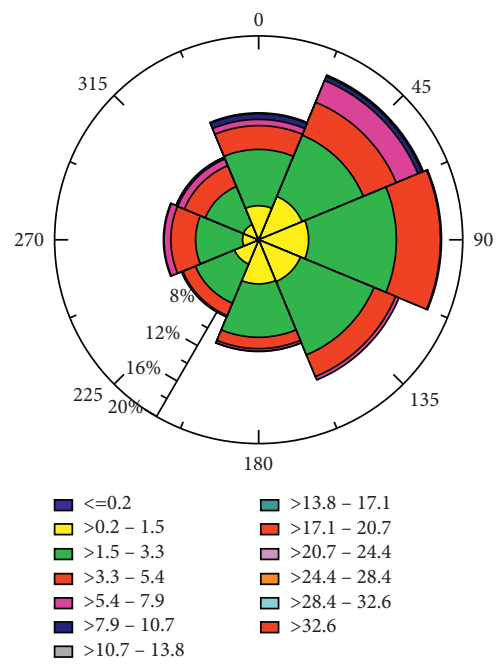

(d)

Figure 2: Meteorological conditions of the Gurbantünggüt Desert in 2017. (a) Air temperature at 2 m. (b) Specific humidity. (c) Air pressure. (d) Wind rise at $10 \mathrm{~m}$.

to the type of sandy soil, the soil surface temperature of the observation site varies greatly. The maximum surface temperature of the soil varies from -8.3 to $55.5^{\circ} \mathrm{C}$, and the lowest surface temperature varies between -34.2 and $13.2^{\circ} \mathrm{C}$. As a desert with certain vegetation, the change in air specific humidity changes with the season and the underlying surface change (Figure 2(b)). The specific humidity of winter is smaller and varies about $1 \mathrm{~g} \cdot \mathrm{kg}^{-1}$. Ephermeral plants bloom and thrive in the spring with a specific humidity of about $5 \mathrm{~g} \cdot \mathrm{kg}^{-1}$ in the ambient air. In the hot summer, due to more precipitation, the specific humidity is about $8 \mathrm{~g} \cdot \mathrm{kg}^{-1}$, while in autumn, the specific humidity is about $3 \mathrm{~g} \cdot \mathrm{kg}^{-1}$. The highest value of air pressure occurred in the winter of December, the lowest value appeared in the summer of June, and the whole year changed between 935 and $981 \mathrm{hPa}$ (Figure 2(c)). In terms of wind speed of $10 \mathrm{~m}$, the most wind direction is concentrated in the east and the northeast, and the most frequent occurrence is in the range $1.5-5.4 \mathrm{~m} \cdot \mathrm{s}^{-1}$ (Figure 2(d)).

According to the statistics of the solar downward shortwave radiation data, the sunrise in January is around 7:30 local time, the sunset is around 17:30 local time, and the average daily sunshine time is about 10.5 hours; the sunrise of April occurred at around 5:30 local time, the sunset is around 19:30 local time, and the average daily sunshine time is about 14.5 hours; the July sunrise time is around 4:30 local time and the sunset time is around 20:30 local time. The average daily sunshine time is about 16.5 hours; the October 
sunrise time is around 6:30 local time, the sunset time is around 18:00 local time, and the average daily sunshine time is about 12 hours.

\subsection{Seasonal Variation of Wind Profiles in the Near-Surface} Layers of the Gurbantünggüt Desert. As shown in Figure 3, under the three underlying surface conditions during spring, summer, and autumn, the changes of the near-surface wind profile were similar during daytime and nighttime. The reason is, on sunny days, there is a temperature inversion phenomenon in the near-surface layer at night, which hinders the development of turbulent motion. Since there is weak turbulent motion, the wind speed increases as the height increases, and as the wind travels up, the speed increases. During the day, due to the Sun's radiation, the nearsurface atmosphere is unstable. As it becomes increasingly unstable, the turbulence increases due to the unstable heating, the wind speed decreases due to the increased interactions between the upper and lower layers, and the wind profile is bent toward the wind speed axis. This finding is the same as that by Miao et al. [17] who performed a study of the wind profile distribution pattern in the atmospheric boundary layer of the southern Xinjiang desert hinterland. Overall, during the night, the wind speed increased as the wind height increased. Thus, the wind speed, wind speed gradient, and the range of changes in wind speed are greater at night than during the day.

During the summer and autumn, the wind speed gradually decreased at 10:00, while during spring, the wind speed continually increased after sunrise. Following the growth of short-lived plants in the spring and herb plants in the summer, the wind speed drag force gradually increased. When the snow melted in the early spring, the lichens and other short-lived vegetation grew abundantly. At night, the wind speed increases by $0.9 \mathrm{~m} \cdot \mathrm{s}^{-1}$ for every $10 \mathrm{~m}$ increase in elevation. During summer, herbs and shrubs grew abundantly, and at night, the wind speed increased by $1.2 \mathrm{~m} \cdot \mathrm{s}^{-1}$ for every $10 \mathrm{~m}$ increase in elevation. During autumn, there is only sand in the desert's underlying surface conditions, and at night, the wind speed increases by $0.8 \mathrm{~m} \cdot \mathrm{s}^{-1}$ for every $10 \mathrm{~m}$ increase in elevation. During the winter, when there was long-term snow cover, the wind speed in the underlying surface was lower than those in the other seasons. During this season, the wind profile was primarily stable at night, and it was inconsistent with the wind profiles of the other three underlying surface types. The wind speed during the day and night increased as the wind moved to higher elevations, and the diurnal variation was not evident. The wind speed increased by $0.4 \mathrm{~m} \cdot \mathrm{s}^{-1}$ for every $10 \mathrm{~m}$ increase in elevation.

\subsection{Seasonal Variation of Temperature Profiles in the Near-} Surface Layers of the Gurbantünggüt Desert. As shown in Figure 4, the temperature profiles under the different underlying conditions exhibited consistent patterns-nighttime radiation, morning transitional, daytime solar radiation, and evening transitional phases. During the different seasons, there were variances in the times when the profiles appeared. This is consistent with the results of the study done by Li et al. [18] on the temperature profile on the Taklimakan central region's near-surface layer on a sunny day.

The morning transitional type profile appeared at 06 : 00-09:00. It appeared earliest during the summer, followed by autumn, and appeared at the latest time during the winter. As the Sun appears and its radiation increases, the surface radiation quickly changes from positive to negative, the ground starts to heat up quickly and disrupts the nighttime inverse temperature in the near-ground layer, and this disruption process gradually moves from the ground's lower layer to the upper layer. As shown in Figure 4(c), during 06:00 in the summer, the profile at a $2 \mathrm{~m}$ height in the near-surface layer exhibits an inflection point. Since the area below the inflection point was affected by the Sun's radiation, it slowly entered the daytime solar radiation type, while the area above the inflection point was still part of the nighttime radiation type. At 08:00 during the summer, the profile already showed signs of being a transitional type, while the profiles of the autumn and winter at this hour were still part of the nighttime radiation type.

During the day, the solar radiation profile appeared at 08:00-20:00. The times when it appeared were as follows (earliest to latest): summer $>$ autumn $>$ spring $>$ winter. The temperature profiles after 14:00 were the same during the summer, spring, and autumn. Since the daytime surface radiation balance was positive, the ground temperature increased rapidly, and the heat was transmitted to the nearsurface layer atmosphere. Therefore, the temperature decreased as the height increased, and at $32 \mathrm{~m}$ in the nearsurface layer, the atmosphere was very hot and unstable (state of superadiabatic instability) (the vertical lapse rate of the air temperature could reach $4.5^{\circ} \mathrm{C} / 100 \mathrm{~m}$ ). As a result of the increased unstable heating, the turbulent mixing increased. Thus, the heat exchange increased between the upper and lower layers, and the heat distribution gradually became uniform. As a result, the speed at which the temperature gradient decreased with height was greater.

The evening transitional profile appeared at 20:00-22:00, and its form was a mix of the solar radiation and radiation types. In the afternoon, it decreased relative to the Sun's height. The surface radiation balance quickly decreased, and the underlying surface cooled quickly, which caused the temperature in the near-surface layer to decrease. However, the upper layer atmosphere was still in a heat preserving state. At this time, the lower layer atmosphere already changed to the evening radiation type, while the upper layer atmosphere was still under the daytime solar radiation type. This characteristic was more evident in the temperature profiles of the spring and summer, while the temperature profiles of the autumn and winter at this time changed to the evening radiation type.

In the winter with stable snow cover, the temperature of the near-surface layer of the desert is below $-15^{\circ} \mathrm{C}$. During the daytime, with the increase of the height of the nearsurface layer, the daily maximum temperature increases, and it is in an inverse temperature state. The vertical rise rate of $0.5 \mathrm{~m} \sim 32 \mathrm{~m}$ is $0.5^{\circ} \mathrm{C} / 100 \mathrm{~m}$. In the spring, summer, and autumn seasons, different from winter, the maximum temperature in the daytime and daytime is obviously 


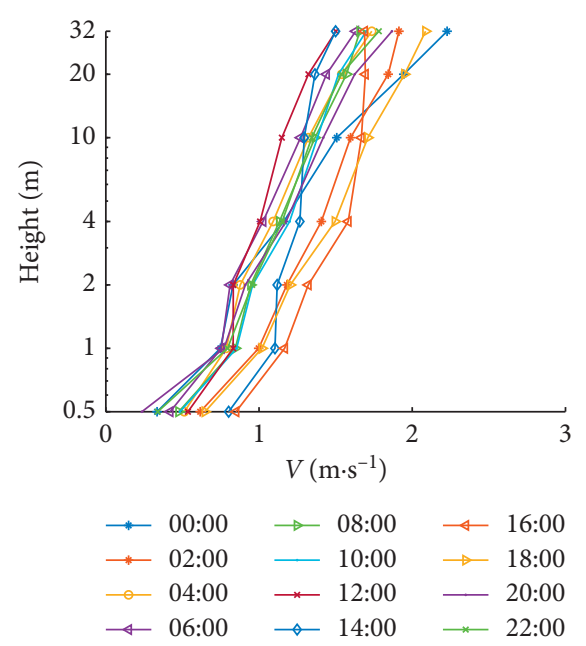

(a)

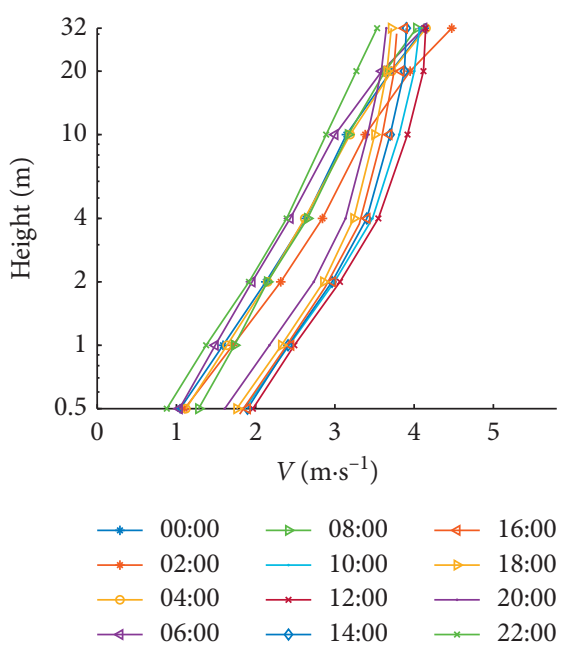

(c)

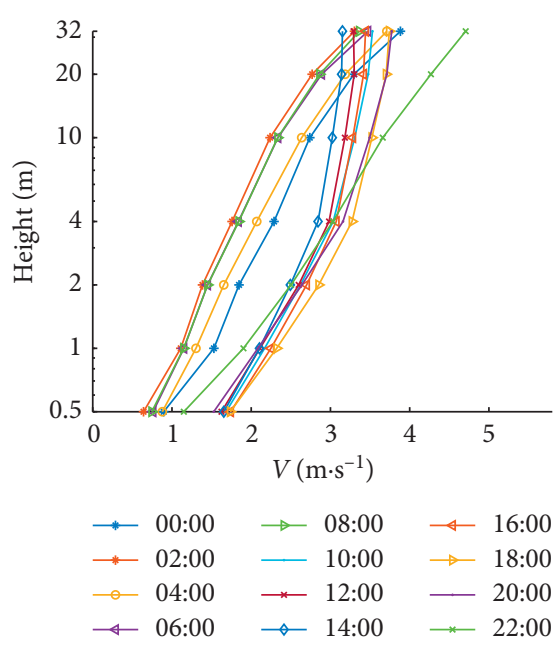

(b)

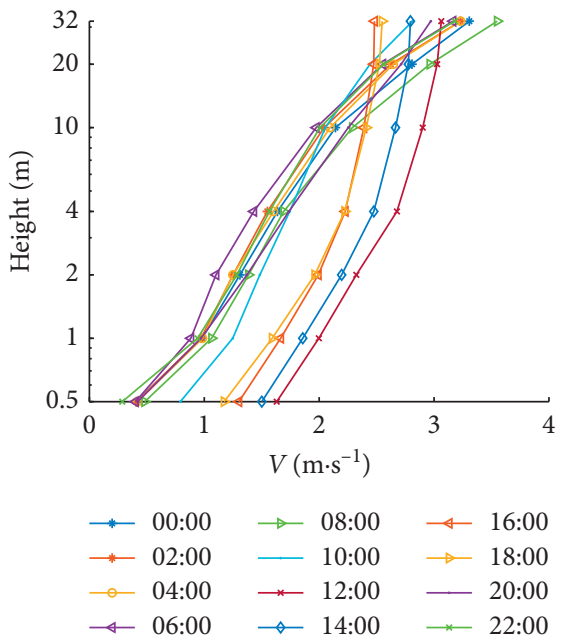

(d)

Figure 3: Wind profiles on sunny day under the four underlying surface conditions in the Gurbantünggüt Desert. (a) Winter. (b) Spring. (c) Summer. (d) Autumn.

weakened with the increase of the height of the near-surface layer. The vertical decline rate of temperature is $0.5^{\circ} \sim 32 \mathrm{~m}$ in the spring and summer, $4.5^{\circ} \mathrm{C} / 100 \mathrm{~m}$ in the spring, and $3.7^{\circ} \mathrm{C} / 100 \mathrm{~m}$ in the autumn. Due to the small specific heat capacity of the sandy soil itself and the climatic characteristics of the desert drought and less rain, the temperature difference between day and night in the desert area is much larger than that of the city, forest, and other underlying surface types. In the four seasons of winter, spring, summer, and autumn, the average daily temperature difference at each height is $6.1^{\circ} \mathrm{C}, 9.2^{\circ} \mathrm{C}, 11.2^{\circ} \mathrm{C}$, and $10.8^{\circ} \mathrm{C}$, respectively. The difference in winter temperature is the smallest in the year, at each altitude. The temperature is continuously reduced from the late $20 \mathrm{~h}$, and the trend of this nighttime cooling is gradually weakened with time. In spring, the temperature difference in the near-surface layer is larger than that in summer and autumn at both the early 06:00 and the late 20: 00 . The overall trend of change is consistent with the seasonal variation of temperature at different heights in spring and the desert regions of Zhu and the desert.
3.4. Specific Humidity Profile in the Near-Surface Layers of the Four Seasons in the Gurbantünggüt Desert. Figure 5 shows the specific humidity profiles on a typical sunny day under the four underlying surface conditions in the spring, summer, autumn, and winter in the hinterland of the Gurbantünggüt Desert. As can be seen, during the spring and summer nights, the specific humidity within $32 \mathrm{~m}$ from the near-surface layer decreased as the height increased, and the vertical lapse rate of specific humidity was slightly higher than that during the day.

During autumn, the specific humidity profile at 10 : 00-16:00 and at heights of 0-10 m was almost equal, while within 10-20 m, the specific humidity decreased as the height increased. From 20:00 in the evening to 08:00 in the morning, and at heights of $2-10 \mathrm{~m}$, the average specific humidity increased as the height increased, creating a humidity inversion state. Compared to the other three seasons, the winter season's specific humidity had a unique characteristic: except for the 10 different hours outside of the 14: 00 and 16:00, the specific humidity increased as the height 


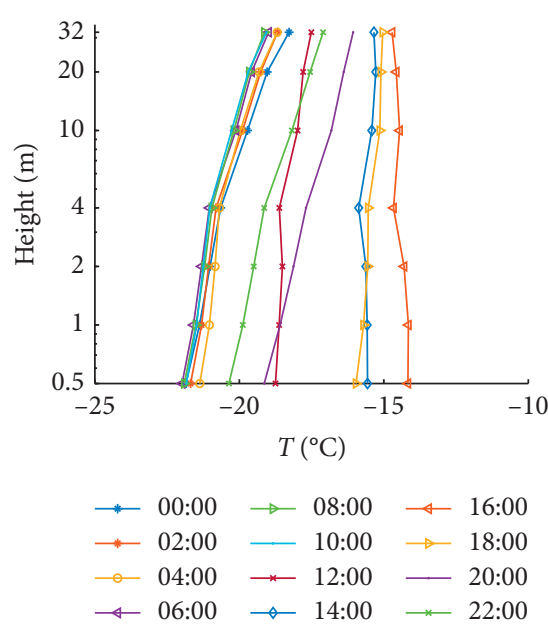

(a)

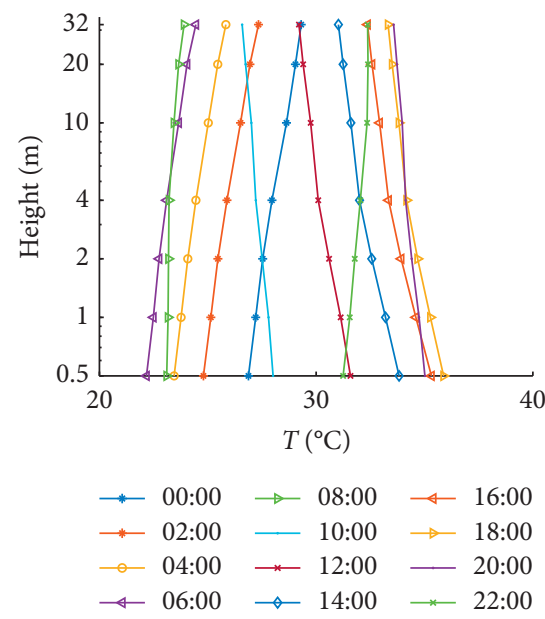

(c)

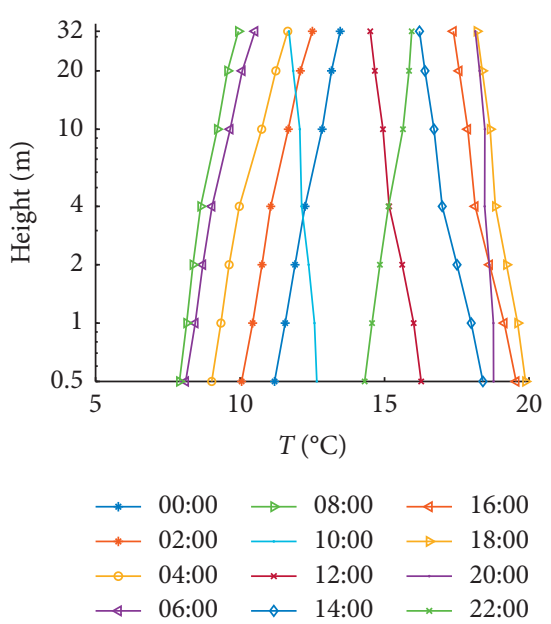

(b)

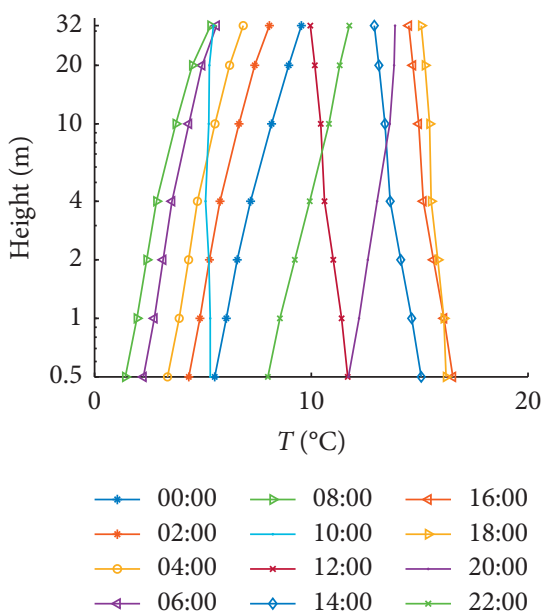

(d)

FIGURE 4: Temperature profiles on typical sunny day under the four underlying surface conditions of the Gurbantünggüt Desert. (a) Winter. (b) Spring. (c) Summer. (d) Autumn.

increased, causing a humidity inversion state distribution. This pattern was different from those of the other seasons.

Specific humidity is affected by meteorological and geographical conditions, among which water vapor transport, ground wind speed and soil humidity, and latitude and longitude positions are the main influencing factors. The specific humidity value in the study area is highest in summer and lowest in winter. This is due to the large number of shrubs growing in summer in the study area, and the precipitation conditions are better than winter. There is no seasonal vegetation, seasonal snow, and ice in the desert in autumn, so it is different from the other three seasons.

\subsection{Discussion on Evolution of Near-Surface Atmospheric} Boundary Layer due to the Underlying Surface Changes. Seasonal changes can cause changes in the near-surface atmospheric boundary layer elements of various regions of the world, including energy and radiation balance. In the context of seasonal changes, due to the difference in the underlying surface or the underlying surface of each place, the situation of near-surface atmospheric boundary layer changes in various places is inconsistent. As the unique desert area of China, the Gurbantünggüt Desert has changed the underlying surface to cause changes in meteorological elements and energy exchange in the near-surface layer. The difference between the Gurbantünggüt Desert and other deserts in northern China is that there is a long period of snow accumulation in winter, and short-lived plants grow and die in spring. The wintertime snow cover in the Gurbantünggüt Desert generally lasts until midMarch. From mid to late April with the conditions of early snowmelt and a small amount of precipitation in spring, there are increases in air and soil temperature, the lichens, and moss on the surface of the Gurbantünggüt Desert and ephemeral plants such as Haloxylon ammodendron grow up, and at the end of May, the ephemeral plants die rapidly.Under the seasonally changing metorological background, the snow cover-bare soil-ephemeral plants switch quickly in the desert surface, which results in changes in the energy budget of the near-surface atmospheric boundary layer altering the boundary layer. In order to explain the change of the underlying near-surface atmospheric boundary layer caused by 


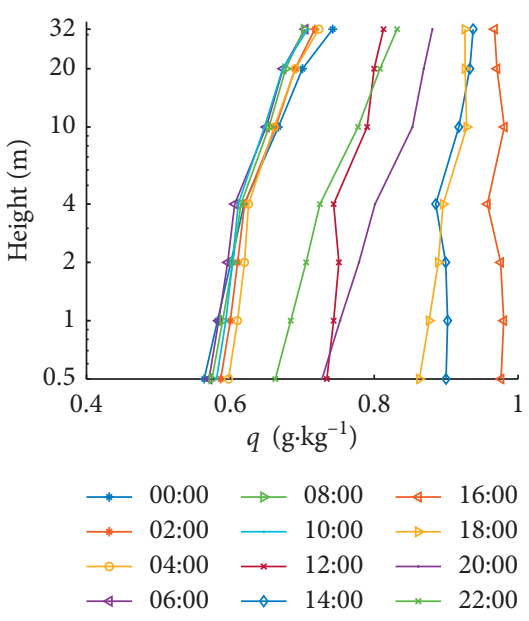

(a)

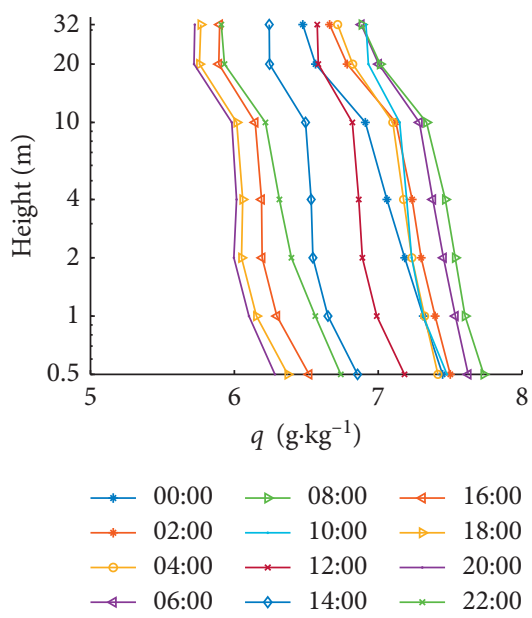

(c)

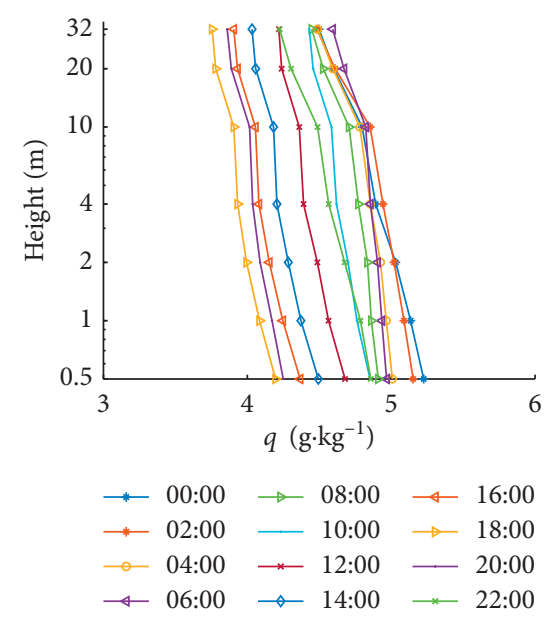

(b)

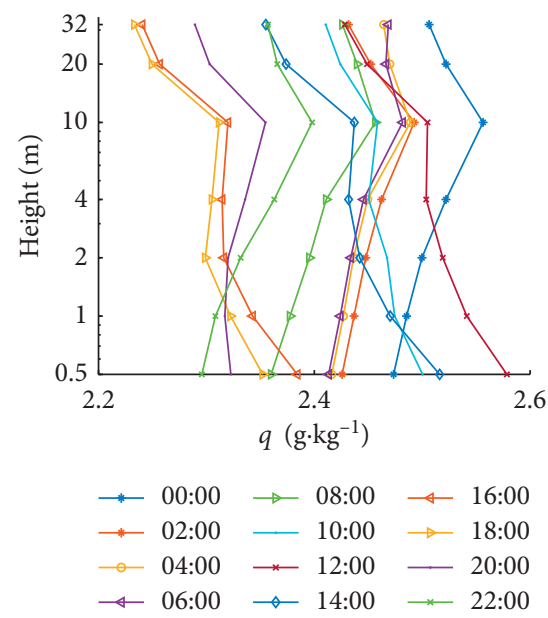

(d)

FiguRe 5: Specific humidity profiles on typical sunny day under the four underlying surface conditions of the Gurbantünggüt Desert. (a) Winter. (b) Spring. (c) Summer. (d) Autumn.

the change of the underlying surface in the background of seasonal changes, this paper selects the flux data corresponding to the sunny days in different periods of 2017, and the satellite data and the whole year meteorological element data were analyzed.

It can be seen from Figures 6(a) and 6(c) that under the effect of winter precipitation, the snow depth of the Gurbantünggüt Desert slowly rises from January to late February, and with the arrival of spring, snow begins to melt in March. On March 23, the snow began to melt rapidly, and on March 25, it reached the peak of melting. On March 27, the snow completely melted. Driven by seasonal changes, the underlying surface of the Gurbantünggüt Desert has changed from a snow cover underlying surface to an underlying surface covered by short-lived plants. The snow that melted in late March quickly penetrated into the desert soil, adding water to the growth of desert short-lived plants. As can be seen from Figure 6(b), the shallow soil moisture began to rise slowly from March 20, accelerated from March 25, reached its highest value on the 27th, and then slowly decreased. The changes in soil moisture not only accelerate the growth of surface vegetation, but also have an effect on changes in soil heat flux. It can be seen from Figure 6(d) that with the increase of air temperature and soil temperature (the soil temperature change map neglected), the short-lived plants such as desert moss and Haloxylon ammodendron grow rapidly under the supplement of previous snowmelt soil moisture and precipitation, and the NDVI index rises to 0.1627 (from Figure 6(d)) in April. In July, under the dual effects of precipitation and temperature rise, shrubs and other vegetation grew in the study area, and the NDVI index rose to 0.1741 . In October, the shrub gradually began to die and the vegetation index dropped to 0.1676 . From the above figure, we can clearly see that in the background of seasonal changes, the change of the underlying surface of the Gurbantünggüt Desert in spring is more "violent" and "faster." This change has an impact on the near-surface atmospheric boundary layer in the desert region, especially on the land-atmospheric interaction. In order to clarify this problem, turbulence flux data of clear sky day representing the different underlying surface periods in the middle of the 

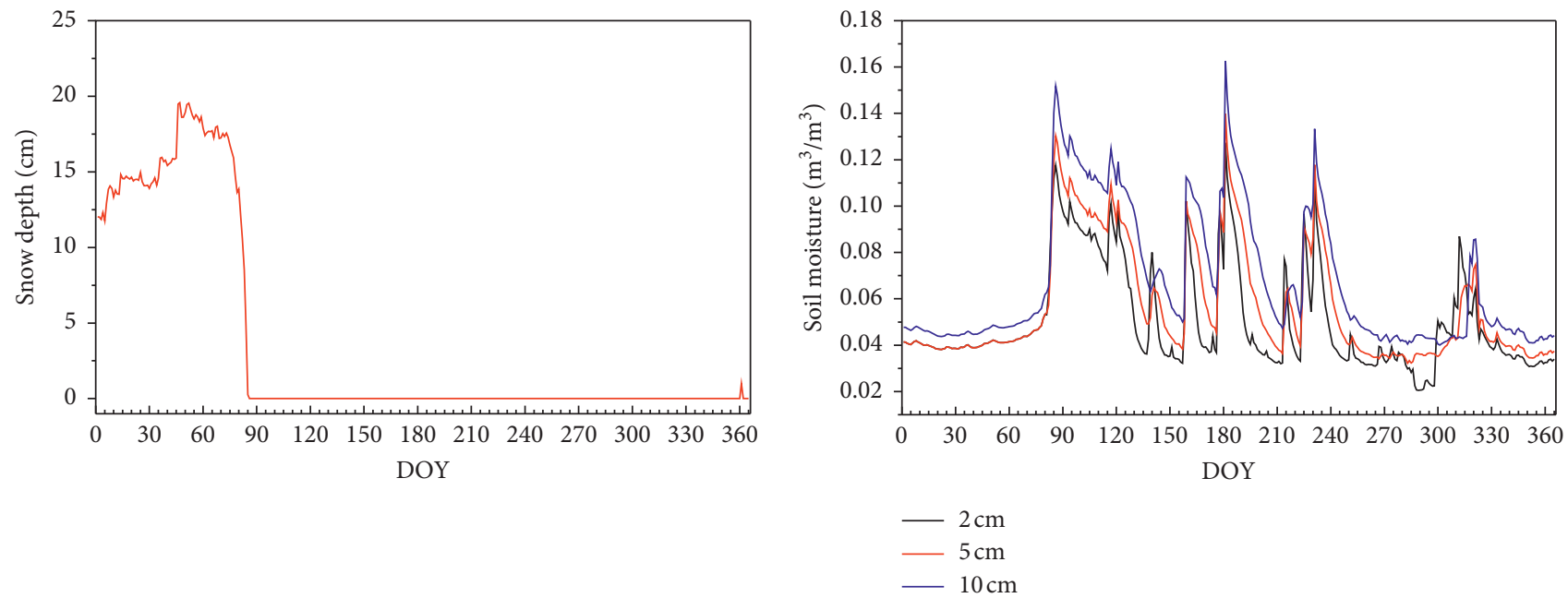

(a)

(b)
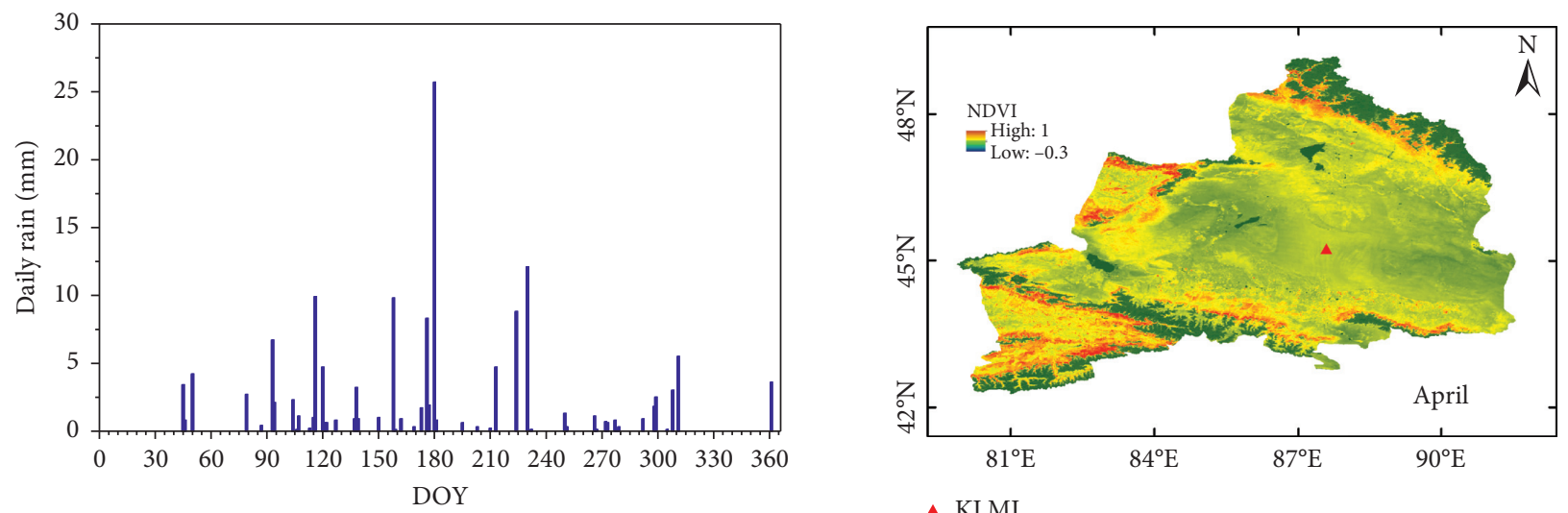

^ KLML

(c)

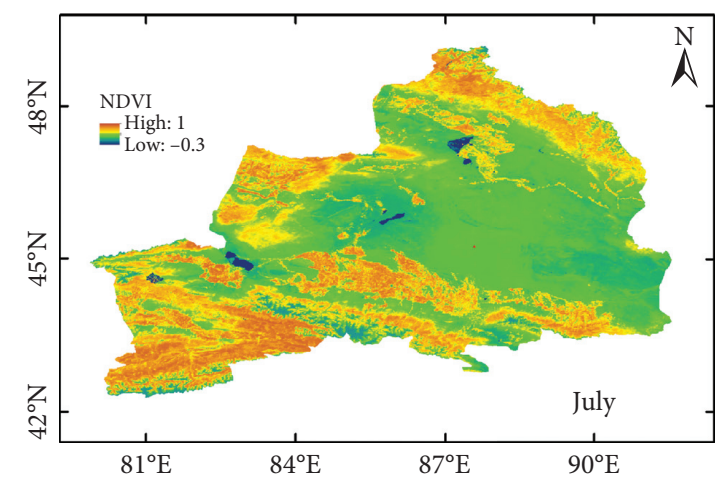

A KLML

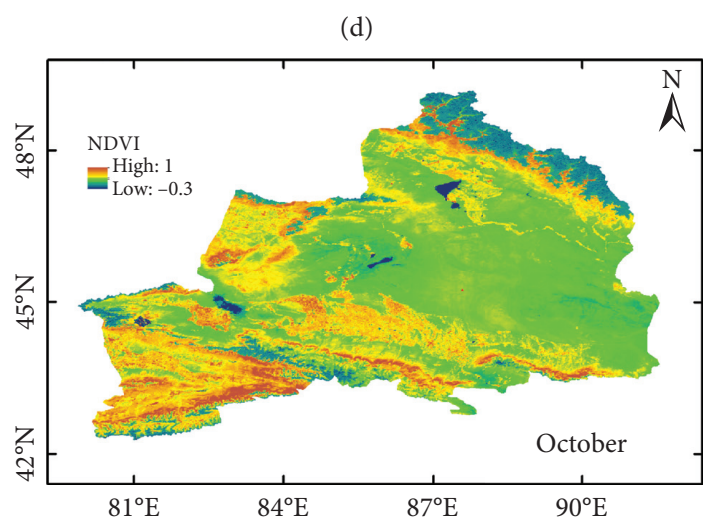

$\triangle \mathrm{KLML}$

(e)

(f)

FIGURE 6: Monitoring of the change of the underlying surface of the Gurbantünggüt Desert in spring. (a) Daily average snow depth. (b) Shallow soil moisture. (c) Daily precipitation. (d-f) Month average NDVI.

3-5 months were selected, and the surface energy balance and energy distribution ratio of the station were analyzed.

It can be seen from Figure 7(a) that under the conditions of snow cover surface in early March, the variation of the surface energy components is small. Daytime net radiation is mainly dissipated in the form of sensible heat flux and soil heat flux. After the snow melted on March 27, the net radiant flux on the surface also increased significantly, and the sensible heat, latent heat, and soil heat flux also increased. This situation continued until the beginning of April. The atmospheric $\mathrm{CO}_{2}$ flux during the same period also showed a weak exchange characteristic. After mid-April, due to the growth of short-lived plants, $\mathrm{CO}_{2}$ exchange increased significantly, especially during the day, and reached a peak in 


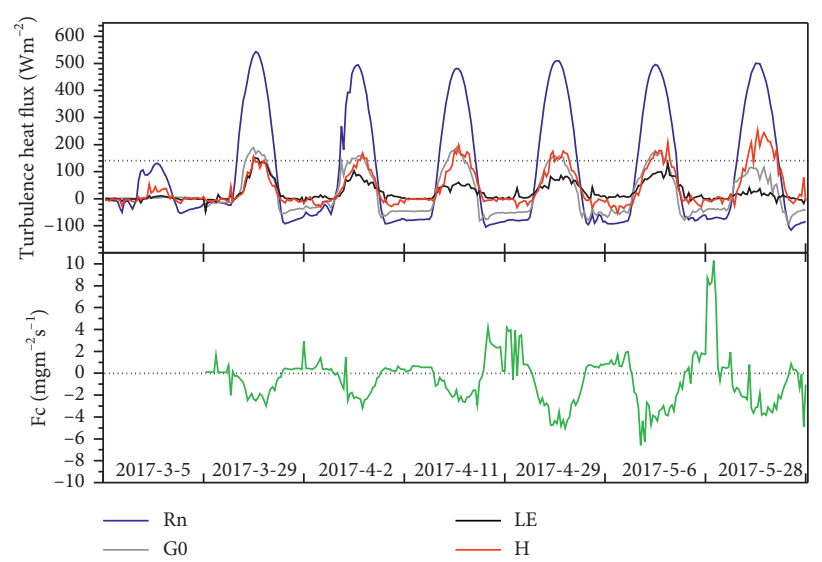

(a)

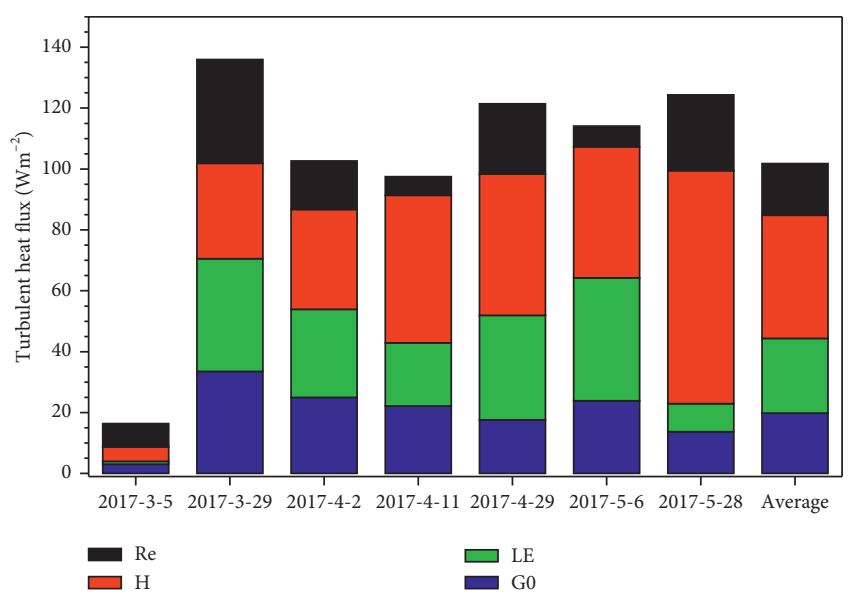

(b)

FIGURE 7: Changes in the surface energy balance of different underlying surface periods. (a) Changes in the components of the surface energy budget. (b) The proportion of the surface energy budget components in the net radiation.

early May. The latent heat flux during the same period has slowly increased. By late May, due to rapid death of ephemeral plants on the surface, the $\mathrm{CO}_{2}$ budgets become smaller. Figure 7(b) shows the proportion change of the daily surface energy budget of the Gurbantünggüt Desert in the daily average net radiation. It can be seen from the figure that under the conditions of snow cover surface, the average daily net radiation is around $20 \mathrm{Wm}^{-2}$, in which the sensible heat flux and the soil heat flux account for a large proportion and the proportion of latent heat flux is small. After the snow melted, the average daily net radiation from late March to early April changed around $100 \mathrm{Wm}^{-2}$. During this period, the latent heat flux in the near-surface atmospheric boundary layer increased significantly due to the effect of snowmelt and precipitation, about $30 \mathrm{Wm}^{-2}$. From midApril to early May, the proportion of latent heat flux increased further due to the rapid growth of short-lived plants. In late May, due to rising temperatures and the gradual death of short-lived plants, the proportion of sensible heat flux in net radiation increased, and the proportion of latent heat flux decreased. From the average situation in the spring, the spring sensible heat flux in the desert hinterland accounted for the largest proportion of net radiation, followed by latent heat flux, and soil heat flux accounted for the smallest proportion. From the above changes in the surface energy budget, it can be seen that in the context of seasonal changes, the "rapid" change of the underlying surface affects the distribution of surface energy budget and the land-atmospheric carbon budget, thus causing the near-surface atmospheric boundary layer change.

In order to understand how changes in the underlying surface affect the near-surface atmospheric boundary layer, we selected data from March to May, July, and October to analyze the evolution of wind and temperature profile. The reason for choosing March to May in spring is that snow cover still exists in the Gurbantünggüt Desert in early March. After late March, the snow quickly melted, and the ground surface became "bare" (including death or dormancy ephemeral plants or soil crusts, etc.). Ephemeral plants grow rapidly from early April to May and die rapidly in late May. This rapid change of the underlying surface in spring provides a very special time for us to study the evolution of the near-surface atmospheric boundary layer caused by the underlying surface change. The shrubs grow rapidly in summer and reach the peak period in July, and the NDVI reaches the highest. Therefore, we chose July data to reveal how the desert shrubs in hot summer affect the near-surface atmospheric boundary layer. We selected October data to reveal evolution of boundary layer change because shrubs tend to die in October.

It can be seen from Figure 8 that under the snow cover situation, due to the high albedo, small available energy received by the ground and the turbulent flow energy are relatively small, mainly due to mechanical turbulence. In this case, the near-surface boundary layer is fully mixed at 14-18 hours during the day, but the inversion layer appears very quickly under the effect of radiation cooling after the sunsets. The height of the near-surface inversion layer is about $10 \mathrm{~m}$ and lasts for 4 hours. At about 6 am, there is an inversion layer with a height of about $2 \mathrm{~m}$. The wind profile changes during the same period are affected by atmospheric stability and turbulent kinetic energy. In the early morning, due to the neutral state of the atmosphere, the wind shear in the near-surface layer is relatively large, and the momentum of the tower is transmitted to the ground. This phenomenon is highest at around $2 \mathrm{am}$. During the daytime between 14 and 16 hours, due to the heating of the ground, the atmosphere is in an unstable state, the vertical motion is relatively strong, the turbulent kinetic energy generated by buoyancy is dominant, and the wind shear in the near-surface boundary layer is small.

The snow in the desert hinterland completely melted in early April, and the underlying surface that was bare but contained dormant or dead ephemeral plants or soil crusts was the main feature of the desert hinterland. In this state, the turbulent kinetic energy is relatively strong at 14:00-16:00, 


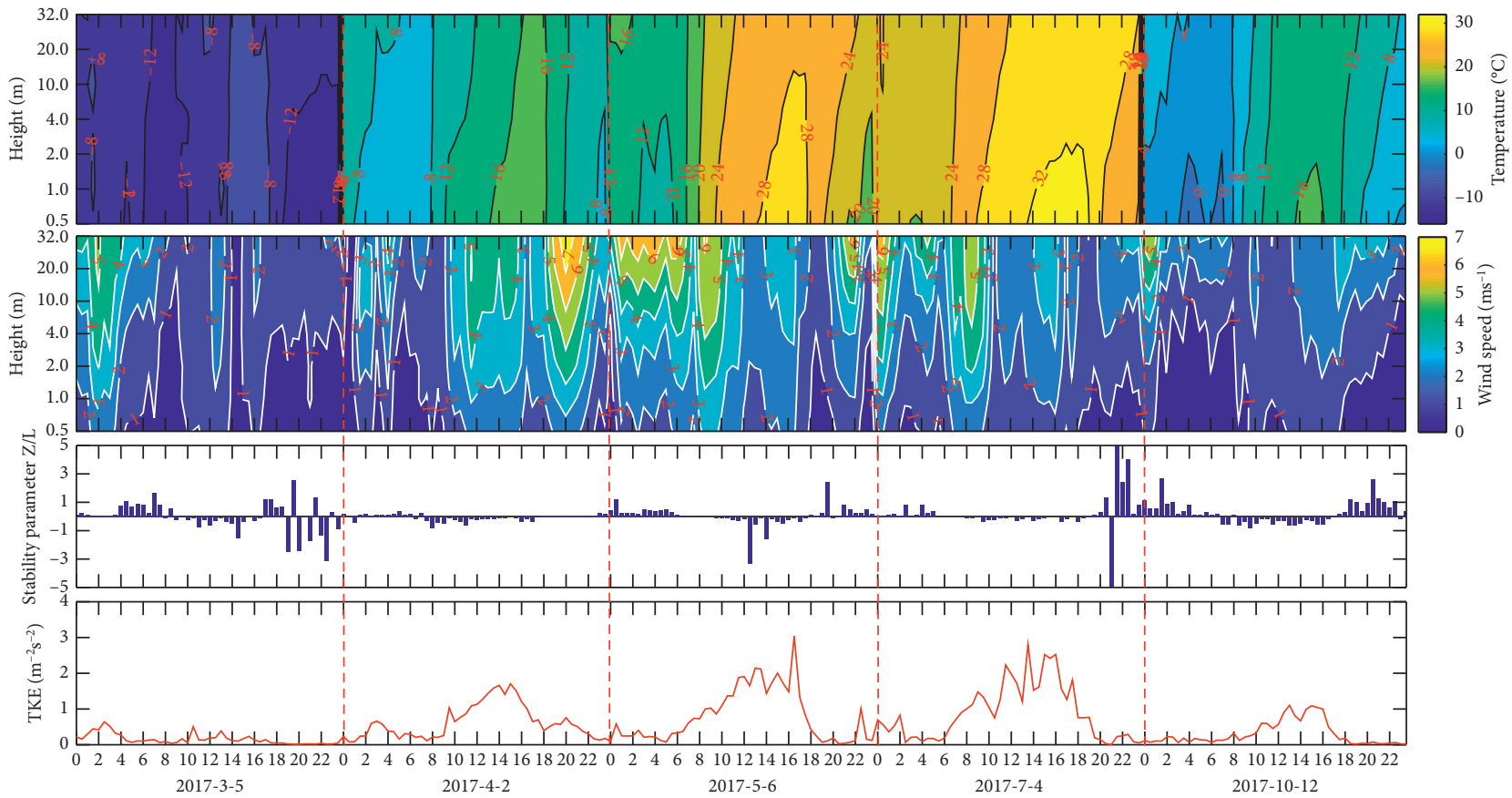

FIGURE 8: Temperature and wind speed profile under different surface conditions.

and the atmospheric instability is relatively strong. The air in the near-surface boundary layer is fully mixed, and there is no inversion phenomenon. Also during this period, the wind profile shear strength is not large. At 14-16 hours, the wind shear strength is relatively large, especially in the evening, when the atmosphere is in a stable state, and the upper and lower wind shears become large. At around 00:00, due to the ground radiation cooling effect, there is an inverse temperature phenomenon, and the inversion temperature of the bottom layer is about $4 \mathrm{~m}$.

At the beginning of May, the growth of short-lived plants in the hinterland of the Gurbantünggüt Desert reached a relatively high level. The entire underlying surface belongs to the underlying surface covered by short-lived plants. In this context, an inversion layer is applied after 10 o'clock at night and around 2 o'clock in the morning. During the daytime, under the condition of strong radiant heating, the upper and lower airflows are mixed, but the phenomenon of complete mixing cannot occur. It can be seen from Figure 8 that under the turbulent kinetic energy generated by buoyancy, a "worm tongue" with a height of about $15 \mathrm{~m}$ appears in the desert hinterland. This is mainly due to the weakening of the atmospheric instability at 14-16 hours, and the turbulent kinetic energy cannot achieve sufficient mixing of the upper and lower air, resulting in the aforementioned "warm tongue" phenomenon. Analysis of the wind profile characteristics shows that the wind-shear strength of the nearsurface boundary layer is enhanced by the influence of the inversion of the night and early morning and the stability of the atmosphere, and the wind shear strength is weakened when the turbulence is strengthened and the instability is enhanced.

In July, the ephemeral plants in the Gurbantünggüt Desert have died, but the shrubs have grown vigorously. Due to the hot summer, turbulent intensity in the desert area is also strong. Whether at night or during the day, the nearsurface atmospheric boundary layer turbulent mixing is sufficient. In this case, there is rarely a near-surface inversion layer or a very weak inversion layer. Due to the strong turbulent intensity during the day, a "warm tongue" with a height of about $2 \mathrm{~m}$ appeared at 13-19 hours, which was obviously inconsistent with the height and duration of the warm tongue that appeared in May. Due to the strong turbulence in summer, the instability of the atmosphere lasts a long time, and the warming of the tongue lasts a long time and wind shear becomes weak. By evening and at night, as the stability of the atmosphere increases, wind shear also increases.

In October, the shrubs in the Gurbantünggüt Desert also gradually began to die, and the turbulence became weaker and the turbulence energy became smaller due to the influence of the change in the height angle of the Sun. Affected by the weakening of turbulence, inversions of varying degrees occur in the desert at night from 2:00 to 8:00 in the morning, especially the strongest inversions around 22:00 at night. Compared to spring and summer, warm tongues appear around 14-16 hours during the day, and the duration and height of warm tongues are shorter and small than in spring and summer. From the profile of wind speed, due to the weak turbulence activity at night, the stability of the atmosphere increases and wind shear also increases.

From the analysis of the temperature and wind profile of the above underlying surfaces, it can be seen that the change of the underlying surface causes the change of turbulent kinetic energy and stability during the daytime, affecting the mixing of the upper and lower air, thereby affecting the change of the near-surface temperature and the wind speed with height. 


\section{Conclusions}

Based on the study and analysis of the wind speed, temperature, and specific humidity on a typical sunny day under the four underlying surface conditions in the near-surface layer of the Gurbantünggüt Desert hinterland, the following can be concluded:

(1) In the near-surface layer of the Gurbantüngüt Desert hinterland, with the underlying surface conditions during the spring, summer, and autumn, the wind profiles can be classified into nighttime stable and daytime unstable types. During the day, the rate of decrease in the wind speed increased with height, and it bent towards the wind speed axis, where the curvature in the spring/autumn was less than that of the summer; at night, the rate of increase in the wind speed was increased with height, and it bent toward the higher axis. In the underlying surface conditions during the winter, when there was long-term snow cover, the wind speed was the lowest, the wind profile type was different from the other three underlying surface types, and the wind profile was more stable at night.

(2) In the near-surface layer of the Gurbantünggüt Desert hinterland, the diurnal variation of temperature on a sunny day under the four underlying surface conditions had visible peak and valley values, and the magnitude of the peaks and valleys and the time of occurrence vary in a different season. At night, due to the radiative cooling, the temperature in the near-surface layer atmosphere increased as the height increased, causing temperature inversion and a stable atmosphere. In summer, the temperature difference between the high and low layers is less than that in autumn and winter. During the spring and summer, the vertical lapse rate of the air temperature could reach $4.5^{\circ} \mathrm{C} / 100 \mathrm{~m}$, while the vertical lapse rate during winter was $0.5^{\circ} \mathrm{C} / 100 \mathrm{~m}$. Thus, the accumulation of snow helped to preserve heat on the near-surface layer. The temperature profiles under the different underlying conditions can be classified as nighttime radiation, morning transitional, daytime solar radiation, and evening transitional phases. In the different underlying surfaces, the times when it appeared were as follows (earliest to latest): summer $>$ autumn $>$ spring $>$ winter. However, the time that the morning transitional profile appeared in the Gurbantünggüt Desert was about $1 \mathrm{~h}$ ahead of the corresponding time in the Taklimakan Desert region [19].

(3) In the near-surface layer of the Gurbantünggüt Desert hinterland, the diurnal variation of the specific humidity in the underlying surfaces during the winter, spring, and summer exhibited visible peak and valley values. During the autumn, the daily variance in the specific humidity was smaller, with no visible extreme value. The change of specific humidity is closely related to the temperature and is related to the near-surface wind speed. Under different underlying surface conditions, the specific humidities in the summer and spring (when there was vegetation) were greater than those in the autumn and winter (when there was snow cover). Compared to the other three seasons, the winter season's specific humidity had a unique characteristic: except for the 10 hours outside of 14:00 and 16: 00 , the specific humidity increased as the height increased, causing a humidity inversion state distribution. During autumn, there was also humidity inversion at night.

(4) In the context of seasonal changes, the "rapid" change of the underlying surface affects the distribution of surface energy budget, the land-atmospheric carbon budget, and turbulent kinetic energy and atmospheric stability, thus causing the nearsurface atmospheric boundary layer change.

\section{Data Availability}

Data used in this paper can be provided by Ali Mamtimin (ali@idm.cn) upon request.

\section{Conflicts of Interest}

The authors declare no conflicts of interest.

\section{Authors' Contributions}

A.M. and W.H. were responsible for conceptualization, investigation, project administration, and funding acquisition. A. M., Y. M., X. Y., F. Y., W.H., and C.Z. curated the data. A.M. and Y.W. were responsible for original draft preparation, visualization, methodology, and validation. A. M., Y. M., and H.S. contributed to formal analysis. A.M., Y.W., and X.Y. reviewed and edited the manuscript.

\section{Acknowledgments}

The authors are very grateful to associate prof. Xinyu Lu from the Institute of Desert Meteorology, CMA, for the help in remote sensing data processing. This research was funded by the National Natural Science Foundation of China (41875023), Central Asia Atmospheric Research Foundation (CASS201711), and Central Scientific Research Institute of the Public Basic Scientific Research Business Professional (IDM2017001).

\section{References}

[1] R. B. Stull, Boundary Conditions and Surface Forcings. An Introduction to Boundary Layer Meteorology, Springer, Berlin, Germany, 1988.

[2] C. Liu, T. Zhao, J. Xiong et al., "A simulated climatology of dust aerosol emissions over 1991-2010 and the influencing factors of atmospheric circulation over the major deserts in the world," Journal of Desert Research, vol. 35, no. 4, pp. 959-970, 2015, in Chinese. 
[3] T. Thomas, Warner. Desert Meteorology, Cambridge University Press, Cambridge, UK, 2004.

[4] L. Zhang and C. Chen, "On the general characteristics of plant diversity of gurbantunggut sandy desert," Acta Ecologica Sinica, vol. 22, no. 11, pp. 1923-1932, 2002, in Chinese.

[5] C. Duan, L. Wu, S. Wang, and L. He, "Analysis of Spatiotemporal patterns of ephemeral plants in the Gurbantünggüt Desert over the last 30 years," Acta Ecologica Sinica, vol. 37, no. 8, pp. 2642-2652, 2017, in Chinese.

[6] M. Chen and S. Niu, "Study on meteorological elements structure of atmospheric surface layer over desert area in spring," Journal of Desert Research, vol. 28, no. 5, pp. 955-961, 2008, in Chinese.

[7] J. Cuesta, D. Edouart, M. Mimouni et al., "Multiplatform observations of the seasonal evolution of the Saharan atmospheric boundary layer in Tamanrasset, Algeria," in The Framework of the African Monsoon Multidisciplinary Analysis Field Campaign Conducted in 2006. Doing Capitalism in the Innovation Economy, Cambridge University Press, Cambridge, UK, 2016.

[8] J. E. Cuesta, J. H. Marsham, D. J. Parker, and C. Flamant, "Dynamical mechanisms controlling the vertical redistribution of dust and the thermodynamic structure of the West Saharan atmospheric boundary layer during summer," Atmospheric Science Letters, vol. 10, no. 1, pp. 34-42, 2010.

[9] M. C. R. Kalapureddy, M. Lothon, B. Campistron, F. Lohou, and F. Saïd, "Wind profiler analysis of the African Easterly Jet in relation with the boundary layer and the Saharan heat-low," Quarterly Journal of the Royal Meteorological Society, vol. 136, no. S1, pp. 77-91, 2010.

[10] L. Garcia-Carreras, D. J. Parker, J. H. Marsham et al., "The turbulent structure and diurnal growth of the saharan atmospheric boundary layer," Journal of the Atmospheric Sciences, vol. 72, no. 2, pp. 693-713, 2010.

[11] C. Messager, D. J. Parker, O. Reitebuch, A. Agusti-Panareda, C. M. Taylor, and J. Cuesta, "Structure and dynamics of the Saharan atmospheric boundary layer during the west African monsoon onset: observations and analyses from the research flights of 14 and 17 July 2006," Quarterly Journal of the Royal Meteorological Society, vol. 136, no. S1, pp. 107-124, 2010.

[12] J. Gu, H. Wen, and Q. He, "Meteorological element profile characteristics of different weather types above two kinds of underlying surface in the north of Taklimakan Desert," Desert and Oasis Meteorology, vol. 8, no. 2, pp. 27-33, 2014, in Chinese.

[13] M. Wang, W. Wei, Q. He, Y. Yang, L. Fan, and J. Zhang, "Summer atmospheric boundary layer structure in the hinterland of Taklimakan desert, China," Journal of Arid Land, vol. 8, no. 6, pp. 846-860, 2017.

[14] Eddypro, 2019, http://www.licor.com/env/products/eddy_ covariance/software.html.

[15] T. Foken, M. Gockede, M. Mauder et al., "Post-field quality control," in Handbook of Micrometeorology: A Guide for Surface Flux Measurements, pp. 81-108, Kluwer Academic, Dordrecht, Netherlands, 2004.

[16] J. Ding, L. Fan, Y. Li et al., "Biomass allocation and allometric relationships of six desert herbaceous plants in the Gurbantünggüt desert," Journal of Desert Research, vol. 36, no. 5, pp. 1323-1330, 2016, in Chinese.

[17] Q. Miao, J. Wang, Q. He et al., “Analysis of meteorological element profile in atmosphere boundary layer in south Xinjiang desert hinterland," Meteorology and Disaster Reduction Research, vol. 32, no. 2, pp. 6-10, 2009, in Chinese.
[18] X. Li, Q. He, M. Ali et al., "Characteristics of temperature, humidity and wind profile in the surface layer in sunshiny day in spring in the Taklimakan Desert hinterland," Ard Land Geography, vol. 31, no. 3, pp. 389-396, 2008, in Chinese.

[19] Q. He, Observational study on boundary layer structure and land-atmosphere interaction in TaZhong of Taklimakan Desert, Ph.D degree dissertation, Nanjing University of Information Science \&Technology, Nanjing, China, 2009. in Chinese. 